\title{
Emergency Caesarean Section in Adolescent Girls at the Referral Health Center in Commune
}

I

\author{
Alassane Traore ${ }^{1 *}$, Mamadou B. Coulibaly' ${ }^{1}$ Soumana 0. Traore ${ }^{2}$, Modibo D. Soumare ${ }^{3}$, \\ Seydou Fane ${ }^{4}$, Bourama Kane ${ }^{5}$, Mamadou Sima ${ }^{6}$, Saliou A. Traore ${ }^{3}$, Seydina A. Beye ${ }^{7}$, \\ Yacouba L. Diallo" ${ }^{8}$, Youssouf Traore ${ }^{4}$
}

${ }^{1}$ Gynecology Service of Hôpital du Mali, Bam, Bamako Mali

${ }^{2}$ Gynecology-Obstetrics Service, Referral Health Center of Commune IV, Bamako Mali

${ }^{3}$ Gynecology-Obstetrics Service, Referral Health Center of Commune I, Bamako Mali

${ }^{4}$ Gynecology-Obstetrics Service University Teaching Hospital (UTH) Gabriel Touré, Bamako Mali

${ }^{5}$ Pediatric Service of Hôpital du Mali, Bamako Mali

${ }^{6}$ Gynecology Service, UTH Point G, Bamako Mali

${ }^{7}$ Anesthesia Resuscitation Service, UTH Point G, Bamako Mali

${ }^{8}$ Medicine and Endocrinology Service, Hôpital du Mali, Bamako Mali

Email: *alassane200@yahoo.fr

How to cite this paper: Traore, A., Coulibaly, M.B., Traore, S.O., Soumare, M.D., Fane, S., Kane, B., Sima, M., Traore, S.A., Beye, S.A., Diallo, Y.L. and Traore, Y. (2020) Emergency Caesarean Section in Adolescent Girls at the Referral Health Center in Commune I. Open Journal of Obstetrics and Gynecology, 10, 298-310. https://doi.org/10.4236/ojog.2020.1020026

Received: January 9, 2020

Accepted: February 22, 2020

Published: February 25, 2020

Copyright ( 2020 by author(s) and Scientific Research Publishing Inc. This work is licensed under the Creative Commons Attribution International License (CC BY 4.0).

http://creativecommons.org/licenses/by/4.0/ (c) (i) Open Access

\begin{abstract}
Teenage pregnancy is common in Mali due to the high rate of unwanted pregnancies and early marriages. The general objective of this study was to study emergency cesarean delivery in adolescent girls at the referral health center (RHC) in commune I of the district of Bamako. It was a descriptive study with prospective data collection from January 1 to December 31, 2018. Any adolescent girls in whom an emergency caesarean section was performed were included. The data were entered and analyzed on SPSS 20.3. Results: we have collected 616 adolescent parturients, 202 caesareans were performed in emergency, with a frequency of $32.8 \%$. The average age was 17 with the extremes of 13 and 19. Early marriage was found out in $95.5 \%$ of our patients, 40 adolescent girls were primiparous (20\%), 4 of whom had an inter-reproductive interval of less than a year. The main indications for cesarean section were fetal distress in 71 cases (35\%), fetal-pelvic disproportion, scar uterus in the border pelvis ( $11 \%$ each), breech presentation in 15 cases (7\%). Anemia (10.4\%) was the main complication, followed by vascular pedicle attack (5.94\%), infection of the operating site (1.5\%) and a bladder wound $(0.99 \%)$. Fetal mortality was $1.4 \%$ and neonatal $3.8 \%$. Conclusion: the association between adolescence and caesarean section in emergency constitutes risk factors which increase maternal and neonatal morbidity and mor-
\end{abstract}


tality. Fighting these factors improves the survival of the mother and the newborn.

\section{Keywords}

Adolescent, Cesarean, Emergency, Mortality

\section{Introduction}

Teenage pregnancy is very common around the world. It poses different problems depending on the cultural, ethnic or religious context [1]. It is at risk of cesarean section by mechanical dystocia [2].

Adolescence is the period of the evolution of the individual from puberty to adulthood. The complexity of the definition justifies the variability of the limit age of adolescence which goes from 10 to 19 years for the World Health Organization (WHO) [3].

In Mali, teenage pregnancy is tolerated culturally and religiously by a large part of the population. The adverse outcomes of these pregnancies and childbirths are mainly: premature delivery, neonatal suffering, the birth of a low-weight fetus and the death of the newborn. The prognosis for early pregnancies often depends on the quality of monitoring of the pregnancy and the skill of the staff during childbirth. Only $6 \%$ of pregnant women in Mali are followed by doctors and 19\% do not perform any prenatal consultation visit [4]. Also, in Mali, the quality of Antenatal Consultation (ANC) and the management of obstetric complications in emergency which are the main pillars of the fight against maternal and neonatal mortality are not, most often, assured in accordance with the standards. We have initiated this study in order to study emergency cesarean delivery in adolescent girls at the Referral Health Center (RHC) in commune I of the District of Bamako.

\section{Patients and Methods}

It was a prospective descriptive study conducted in the department of gynecology of the referral health center (RHC) of Commune I in Bamako from January 1 to December 31, 2018. Any adolescent girl in whom an emergency Cesarean section was performed was included. According to WHO definition, we have retained adolescent as a girl aged between 10 to 19 years. For each patient, an individual survey sheet was established containing the following variables: the socio-demographic characteristics of the patient; circumstances of admission, medical and surgical history, history of pregnancy, clinical examination; indications for cesarean section; the surgical act; prognostic factors, APGAR; anthropometric measurements, the fate of the newborn. Data collection was done from the prenatal consultation booklet, obstetric records; the registers of operating report, anesthesia, delivery, the partograph, the Reference/Evacuation sheet. The data was entered and analyzed on SPSS 20.3. 


\section{Results}

We have collected 616 cases of teenage deliveries and performed 202 emergency caesareans, with a frequency of $32.8 \%$. The average age of the patients was 17 with the extremes of 13 to 19 years. They were in the early adolescence (10 to 14 years) 11 in number (5.4\%) (Table 1 ).

The patients who were not living in the health area where the study was conducted were forty (40) or 19.8\%. Married adolescents were 193 (95.5\%), out of whom two were under the age of 14 . religious marriage was found out in $125 \mathrm{pa}-$ tients (64.8\%). The mode of admission was self-referencing in 129 cases (63.9\%). Primigravida were 121 (79.7\%). Those out of school were 104 (51.5\%). Of 192 patients (95.04\%) who did antenatal consultations (ANC), 152 (75.2\%) were followed by midwives. The patients who have done four or more prenatal visits were $90(44.5 \%)$ and $10(5 \%)$ had not performed any prenatal consultation (Table 2).

No prenatal check-up was carried out in 32 adolescent girls (15.8\%). On admission examination, an abnormality of fetal heart noise was observed in 66 patients (32.7\%), resulting in bradycardia in 60 cases (29.7\%), tachycardia in 3 cases $(1.5 \%)$ and the absence of fetal heart noise in 3 cases (1.5\%). The breech and transverse fetal presentations accounted for 25 cases (12.4\%) and 4 cases (2\%) respectively. The water bag was broken in 167 patients $(82.7 \%)$. The vaginal examination had found a border pelvis in 78 cases (38.6\%), narrowed in 24 cases (11.9\%). The amniotic fluid was stained in 75 patients (44.9\%). During the surgical intervention, 2 cases of bladder wound were provoked and 12 cases (5.94\%) of vascular pedicle involvement. The transfused patients were $24(11.9 \%)$ of whom 12 (5.9\%) had blood loss greater than $1000 \mathrm{ml}$ during cesarean section. General anesthesia was performed in 116 cases (57.4\%). Postoperative complications were observed in 24 patients (11.88\%) including 3 cases of parietal suppurations $(12.5 \%)$. The number of newborns who were born from caesarean sections was 213 , stillbirths 3 or $1.3 \%$, an APGAR $\leq 7$ was noted in 68 newborns (31.9\%). Macrosomia with a birth weight $\geq 4000 \mathrm{~g}$ accounted for 16 cases (7.5\%).

Table 1. Distribution of patients according to age.

\begin{tabular}{ccc}
\hline Group age & Number & Percentage \\
\hline 10 to 14 years & 11 & 5.4 \\
15 to 19 years & 191 & 94.6 \\
Total & 202 & 100 \\
\hline
\end{tabular}

Table 2. Distribution of healthcare providers who have followed pregnancy.

\begin{tabular}{ccc}
\hline healthcare providers who have followed pregnancy & Number & Percentage \\
\hline Doctor & 40 & 19.8 \\
Midwife & 152 & 75.2 \\
Total & 192 & 100
\end{tabular}


Among the newborns 71 (33.3\%) were resuscitated, 99 (46.5\%) were evacuated in pediatrics. We have not recorded any maternal death but 8 neonatal deaths were reported (3.8\%).

\section{Comments and Discussion}

The frequency: 616 adolescent girls gave birth during the study period, including 202 by caesarean (32.8\%). Childbirth of adolescent girls in Mali is common due to customary marriages which do not take into account the age of the young girls but rather the physical build and menarche. These practices persist despite the Family Act in Mali that sets the age of marriage for girls at 16 . The frequency of adolescent cesarean section in our study (32.8\%) was lower than that of Sawadogo Y A [5] who found 56.4\%. This difference could be accounted for by the fact that in our study adolescent girls with serious pathologies associated with pregnancy were evacuated to hospitals. In Mali, referral health centers are at the second level in the health pyramid, these centers do not have resuscitation services and the treatment of pathologies such as placental abruption, eclampsia is not provided there.

Age: The youngest patient was 13 years old, and ten others were 14 years old. These patients were neither physically nor mentally mature. Early adolescence corresponds to the period of development and maturation of organs including the pelvis. Immaturity of the pelvis can cause serious consequences during childbirth. The lack of education of young teenage mothers is a real obstacle to the fulfilment and development.

Residence: Each health center covers a well-defined geographic area called "the health areas". Referral health centers have ambulances that only transport patients in emergency situations in a health facility which is part of its health area. $20 \%$ of our patients were living outside the health area and had to go to the care facility by their own means of transport. They often arrived in public transport vehicles or on mopeds. This causes not only a delay in joining health centers but also can worsen certain diseases such as placenta previa, retro placental hematoma. The accessibility of health care centers remains an obstacle in the management of obstetric emergencies.

Marital status: Single adolescent girls accounted for only $4.5 \%$, among them 95.5\% were married. Dembélé S and Souley I found $79.2 \%$ and $77.3 \%$ respectively.

The Family Act in Mali sets up 16 the age of marriage for young girls. This legal provision is circumvented by religious and customary marriages. Marrying young girls in adolescence is a way for families to avoid any risk of humiliation. To see a young girl pregnant out of wedlock is a shame for families in Mali.

Occupation: housewives were the most numerous $(74.8 \%)$. Without financial resources to meet families' needs, women in general and adolescents in particular are very fragile.

Level of education: the level of education is very low. Only one adolescent 
girl in five were educated at the beginning of their pregnancy. Adolescents out of school represented 51.5\%. In France Faucher [6] observes that $45.2 \%$ of pregnant adolescents are not in school, Combes [7], in Réunion, finds $47 \%$. Several studies have underlined the importance of schooling in preventing the phenomenon of early pregnancy. In the United States, adolescent girls in school under the age of 12 are 6 times more likely to become mothers before the age of 18 [8].

Mode of admission: $63.9 \%$ of our patients came by themselves, this does not permit insight into the delay in transport since the departure time is not known by the patients. Diallo BS, in his study, found out $85.4 \%$ of patients who had come by themselves.

Surgical history: the risk of complications of pregnancy and childbirth is greater in the case of a scar uterus. $16.33 \%$ of adolescent girls had a scar uterus, they were at risk for delivery dystocia and hemorrhage from delivery. Among them, 4 patients had performed no prenatal consultation.

Gravidity: $20.30 \%$ were pauciparous, $9 \%$ had a spontaneous abortion at the first pregnancy. Whatever is the outcome of a pregnancy, a recovery period is necessary to consider another pregnancy.

Parity: in our series $19.8 \%$ of adolescent girls were primiparous. Dembélé F found $25 \%$ in 2015.

Monitoring of pregnancy: prenatal consultation is a key element for a positive experience of pregnancy. $5 \%$ of our patients had not performed any prenatal consultation, this rate is comparable to that of Drabo A [9] 6.8\%. Several studies agree that the quality of pregnancy monitoring is significantly lower in adolescent girls. They receive fewer consultations than their elders and the average term of the first ultrasound is delayed [9]. Most authors also observe a poorer follow-up in adolescent girls [10] [11] as well as a later start of medical surveillance of their pregnancy [12]. The younger the adolescent, the later the onset of pregnancy monitoring and the poorer the quality of the monitoring [13]. More specific reasons for adolescent girls, such as the fact that pregnancy is not wanted or that it is hidden from those around them, contribute to poor monitoring of their pregnancies.

$50.5 \%$ of adolescent girls in our study had done less than four antenatal consultations, Drabo A in Mali [9] found 40.6\%, Prosper KL in the DRC 31.8\% [14]. Health providers do not perceive adolescent pregnancy as a high-risk pregnancy. Prenatal adolescent consultations are done at the same rate as safe pregnancies. In Mali, in the documents of standards and procedure policies, the number of antenatal consultations in the absence of proven risk is four.

Nowadays the WHO recommends at least eight contacts with the pregnant woman. This concept must be taught to the health providers especially the midwives who assure the majority of the prenatal consultations. In Mali standards, midwives have to monitor pregnancies without pathologies and refer pregnancies at risk.

In our study $75.2 \%$ of pregnancies were followed by midwives. In the litera- 
ture, we did not find information on the qualification of health providers who monitor adolescent pregnancy. However, pregnant adolescents should be followed by gynecologists to identify indications for cesarean section and better programming in order to avoid the urgency which increases maternal and fetal morbidity and mortality.

Prenatal check-up: adolescent pregnant women who had not performed any prenatal check-up were $15.8 \%$. This is likely to increase comorbidity and delay treatment when facing with certain emergencies.

Pathologies occurring during pregnancy: according to several authors, the young age associated with pregnancy exposes to more complications.

Anemia: In Africa, the effects of malaria, parasitic infections of iron deficiency combine to give anemia in pregnant women. Anemia seems more marked in adolescent girls, probably due to the poor follow-up of pregnancy.

We were unable to establish the anemia rate on the basis of blood count because very few of our patients had it. However, 5.9\% of our patients had pale conjunctiva.

Blood pressure: pregnancy-induced hypertension ranges from $3.5 \%$ according to Hardy [15] to $21.9 \%$ according to Janky. $2.5 \%$ of our patients had hypertension whereas many studies find a high frequency of hypertension in adolescent girls. [2]. There is a high prevalence of pregnancy toxemia which we could not analyze correctly because they did not meet the inclusion criteria of our study. Such cases are evacuated to suitable management structures.

Noise of the fetal heart: health facilities in Mali do not have a cardiotocograph for monitoring during labor, listening to noise from the fetal heart is a very important element of fetal well-being. Bradycardia or fetal tachycardia causes fetal distress and forces obstetricians to extract in emergency. We found $1.5 \%$ tachycardia and $29.7 \%$ fetal bradycardia which have a definite influence on the survival of the newborn. $1.5 \%$ of parturients had no fetal heart noise at the time of admission to the delivery room.

Presentation of the fetus: All the patients whose fetus had another presentation other than that of the summit should have received a prophylactic cesarean allowing to reduce the maternal and fetal morbidity related to the emergency context of the cesarean. The breech and transverse presentations represented $12.4 \%$ and $2 \%$ respectively.

The state of the water bag: the time it takes to open the water bag determines the possibility of maternal and or neonatal infection. In our study, $82.7 \%$ of patients were admitted with the broken water bag and $98.2 \%$ had a rupture time of less than 6 hours. Drabo A and Hamada $\mathrm{H}$ found out $19.1 \%$ and $21.1 \%$ of rupture of the water bag. These rates, significantly lower than ours $(82.7 \%)$, are explained by the fact that we were simply interested in the emergency cases whereas Drabo and Hamada have included all emergencies or cold deliveries regardless of the route of delivery.

The aspect of amniotic fluid: we found out $44.9 \%$ of patients who had a me- 
conial amniotic fluid against $4.3 \%$ in the series of Drabo. Frequent scheduled cesareans in the study of Drabo were not included in our work. In the absence of fetal monitoring, the tinted coloration of the amniotic fluid is an important element that alerts the practitioner.

The obstetric pelvis: in adolescents, the pelvis grows more slowly and gradually until old age [14]. In addition, the acquisition of adult size does not imply equivalent growth of the pelvis because "the pelvis does not definitively finish its configuration until around the $25^{\text {th }}$ year albeit the adult forms are reached around the age of 16 " [14].

The patients in our series had an abnormal pelvis in 50.5\%. Adolescent girls with immature pelvis with no prenatal visits were $2.9 \%$, followed mostly by midwives $86 \%$ and only $10.78 \%$ by doctors. Prenatal follow-up of high-risk pregnancies must be done by gynecologists and we classify adolescent pregnancy in this category.

Intraoperative incidents: the urgent context of the caesarean section increases the risk of accident during the surgical intervention. In our study, the cases of bladder wound were $0.99 \%$ and of vascular pedicle attack $5.94 \%$. This latest incident is responsible for a significant loss of blood. A blood loss of more than $1000 \mathrm{cc}$ during the caesarean is considered a hemorrhage of the delivery.

Blood transfusion: it results from an anemia situation aggravated by pregnancy and childbirth, from a hemorrhagic pathology during pregnancy, labor or in the postpartum period. A blood transfusion can occur after a lesion of the uterine pedicle during the cesarean section. $11.9 \%$ of the patients in our series were transfused.

Post-operative complications: in the general population, post-cesarean complication rates in adolescent girls are not different from those in adults, nevertheless, the urgent context of cesarean section can be avoided with appropriate follow-up. $12.5 \%$ of the patients in our study had an operating site infection.

The newborn: some authors observe a higher rate of prematurity in teenage pregnancies. Our results do not seem to be in favor of such a risk.

The weight of the newborns in our series was mainly between $2500 \mathrm{~g}$ and 3500 $\mathrm{g}(89.2 \%)$. Some studies find a lower average weight of newborn babies for teenage mothers compared to adult mothers. Macrosomia with a birth weight greater than $4000 \mathrm{~g}$ was found in $7.5 \%$ of our patients. Dembélé $\mathrm{F}$ and Drabo A found $1.02 \%$ and $0.2 \%$ respectively. We did not find a rational accounting for the high macrosomia rate in our work.

The percentage of depressed infants with an APGAR score $\leq 7$ at the $5^{\text {th }}$ minute was $31.9 \%$. This rate is higher than that of Prosper $\mathrm{K}$ who pointed out $8.2 \%$. This is explained by the difference in methodology between the studies. We have included obstetric emergencies with caesarean section as a delivery route whereas in the study of Prosper the vaginal and high delivery are included with or without emergency.

Evacuation in neonatology: According to Mali standards and procedures 
policies, any newborn who have undergone resuscitation in the delivery room have to be evacuated to the pediatric service. $46.5 \%$ of the newborns in our study have been transferred to pediatric department.

Death: we have not recorded any maternal deaths. However, eight newborns were reported died.

\section{Conclusion}

Emergency cesarean section in teenage is a source of stress for the patient, parents and medical staff. It did not pose any major problems with regard to its completion and the execution time. Better monitoring of pregnancies can permit to reduce the number of emergency caesarean sections and decrease maternal and neonatal morbidity and mortality.

\section{Conflicts of Interest}

The authors declare no conflicts of interest regarding the publication of this paper.

\section{References}

[1] Teagle, S.E. and Brindis, C.D. (1998) Substance Use among Pregnant Adolescents: A Comparison of Self-Reported and Provider Perception. Journal of Adolescent Health, 22, 229-238. https://doi.org/10.1016/S1054-139X(97)00170-5

[2] Iraqi, B., Knouni, H. and Barkat, A. (2017) Pregnancy and Childbirth in the Adolescent Girl: Characteristics and Profile with Regard to 308 Cases. http://aplf2013.com/j print17/grossesse $\% 20$ et $\% 20$ accouchement $\% 20$ chez $\% 20$ adoles cente.pdf

[3] WHO (2017) Adolescents: Health Risks and Solutions. http://www.who.int/mediacentre/factsheets/fs345/fr

[4] Institut National de la Statistique (INSTAT), Cellule de Planification et de Statistique Secteur Santé-Développement Social et Promotion de la Famille (CPS/SS-DS-PF) et ICF. 2019. Enquête Démographique et de Santé au Mali 2018. Bamako, Mali et Rockville, Maryland, USA: INSTAT, CPS/SS-DS-PF et ICF. 2018 Demographic and Health Survey

[5] Sawadogo, Y.A., Ouédrago, I., Zamané, H., Ouattara, A., Kiémtoré, S., Kain, P., Millogo, F. and Thieba, B. (2016) Emergency Cesarean in Adolescents in the Gyn-Obstetrics Department of UTH-YO: Frequency, Indications, Maternal and Perinatal Prognosis. 4th Congress of the Society of Gynecology and Obstetrics of Cote d' Ivoire, Yamoussoukro, 9-12 September 2017, 82.

[6] Faucher, P., Dappe, S. and Madelenat, P. (2002) Maternity in Adolescence: Obstetric Analysis and Review of the Influence of Cultural, Socio-Economic and Psychological Factors from a Retrospective Study of 62 Files. Gynécologie Obstétrique \& Fertilité, 30, 944-952. https://doi.org/10.1016/S1297-9589(02)00497-6

[7] Combes, J.C. and Reynaud, B. (1988) Adolescence and Maternity in Reunion. Pediatrics in Review, 24, 261-264.

[8] Taylor, D., Chavez, G., Habra, A. and Boggess, J. (1997) Risk Factors for Adult Paternity in Births to Adolescents. Obstetrics \& Gynecology, 89, 199-205. https://doi.org/10.1016/S0029-7844(96)00481-4 
[9] Drabo, A. (year) Teenage Pregnancy and Childbirth at the Referral Health Center in Commune II of the District of Bamako.

[10] Soula, O., Carles, G., Largeaud, M., El Guindi, W. and Montoya, Y. (year) Teenage Pregnancies and Childbirth under 15. Study of 181 Cases in French Guyane. Department of Gynecology-Obstetrics, Hospital Center of West Guyane Franck Joly, Saint-Laurent-du-Maroni.

[11] Fraser, A.M., Brockert, J.E. and Ward, R.H. (1995) Association of Young Maternal Age with Adverse Reproductive Outcomes. The New England Journal of Medicine, 332, 1113-1117. https://doi.org/10.1056/NEJM199504273321701

[12] Janky, E., Gallais, A. and Landre, M. (1996) General Information on Teenage Pregnancy and Childbirth. Surg. Med. Encycl., Elsevier, Paris, Gynecology/Obstetrics, 5-016-D-10, 6 p.

[13] Chang, S.C., O’Brien, K.O., Schulman, N.M., Mancini, J. and Witter, F.R. (2003) Characteristics and Risk Factors for Adverse Birth Outcomes in Pregnant Black Adolescents. The Journal of Pediatrics, 143, 250-257. https://doi.org/10.1067/S0022-3476(03)00363-9

[14] Luhete, P.K., et al. (year) Study of the Maternal and Perinatal Prognosis during Childbirth in Adolescent Girls in Lubumbashi, Democratic Republic of Congo.

[15] Hardy, J.B., King, T.M. and Repke, J.T. (1987) The Johns Hopkins Adolescent Pregnancy Program: An Evaluation. Obstetrics \& Gynecology, 69, 300-306. 


\section{Appendix: Individual Survey Sheet}

Theme: Emergency Caesarean section in adolescents: frequency, indications, maternal and perinatal prognosis at the reference health center in commune I of the district of Bamako.

(Q1) DATE: ..../..../ 201...

(Q2) Sheet No. / I

I-Sociodemographic characteristics

(Q3) Last name / . / /

(Q4) First names / .

(Q5) Age / / years

(Q6) Residence / / $(1$ = Commune I, 2 = Outside Commune I $)$

(Q7) Ethnic group / / ( 1 = Bambara, 2 = Peulh, 3 = Soninké, $4=$ Malinké, 5 = Sénoufo, $6=$ Minianka, $7=$ Bobo, $8=$ Khasonké, $9=$ Moorish, $10=$ Sonrhaï, $11=$ Dogon 12 = Moor, $13=$ Dafing, $14=$ Bozo, $15=$ Other $)$

Q8) Marital status / / (1 = Married, 2 = Single $)$

$\mathrm{Q}$ (8a) If married, type of marriage: / / $(1$ = Civil marriage, 2 = Religious marriage)

(Q9) Profession / / ( 1 = Housewife, 2 = Employee, $3=$ Trader, $4=$ Pupil, $5=$ Student, 6 = Other)

(Q10) Level of education / / (1 = Primary, $2=$ Secondary, $3=$ higher, $4=$ Not educated)

(Q11) Nationality / / $(1$ = Malian, 2 = Burkinabé, $3=$ Ivorian, $4=$ Nigerian, 5 = Senegalese, $6=$ Mauritanian, $7=$ other $)$

II-circumstance of admission:

(Q12) Mode of admission / $/(1=$ self-appointed, $2=$ referred, $3=$ evacuated)

(Q13) Addressed by / / ( 1 = doctor, 2 = midwife, $3=$ nurse, $4=$ herself $)$

(Q14) If evacuated / referred, structure of provenance / / $(1$ = Doctor's office, 2 = Medical clinic, 3 = Community Association Health Center (CAHC), 4 $=$ Community Health Center $(\mathrm{CHC}), 5=$ Confessional center $)$

(Q15) Reasons / / $(1=$ Procidence of the cord, $2=$ Breech in primigravida, 3 = Acute Fetal Distress, 4 = Boundary pelvis, 5 = stationary dilation, $6=$ Pelvis generally narrow (PGN) in labor, $7=$ excessive uterine height, $8=$ transverse presentation, $9=\mathrm{HBP}, 10=$ Pre-eclampsia, $11=$ Eclampsia, $12=$ Bleeding on pregnancy, 13 = Placenta Prævia, $14=$ Retro-Placental Hematoma, $15=$ Painful uterine contractions (PUC), $16=$ Vicious presentation, $17=$ Scar uterus, $18=$ Loss of water, $19=$ Twin pregnancy on scar uterus, $20=$ PRM on scar uterus, 21 = Twin pregnancy with $\mathrm{T} 1$ in breech presentation, $22=$ Immature pelvis, 23 = Prolonged expulsive phase, $24=$ Failure to engage, $25=$ Lack expulsive effort, 26 = Refusal to push, 27 = Refusal to cooperate, 28 = overdue delivery, $29=$ Other)

III-History

(Q16) Medical: / / $(1=$ None, $2=$ HBP, $3=$ Diabetes, $4=$ Asthma, $5=$ 
Sickle cell anemia $6=$ Heart disease, $7=$ Other)

(Q17) Surgical: $(1=$ None, $2=$ Cesarean section, $3=$ Myomectomy with opening of the uterine cavity, $4=$ Ruptured uterus, $5=$ Other)

(Q18) Obstetric:

-Gravida: $(1=$ Primigravida, $2=$ Paucigravida, $3=$ Multigravida, $4=$ Large multigravida $)$-Parity: $(1=$ Nulliparous, $2=$ Primiparous, $3=$ Pauciparous, $4=$ Multiparous)

(Q19) Inter-reproductive interval/ / in years

IV Risk factors:

1-History of pregnancy:

(Q20) Term pregnancy / $/(1=$ Yes, $2=\mathrm{No})$

(Q21) followed by / $/(1=$ doctor; $2=$ midwife, $3=$ other person, $4=$ not followed)

(Q22) Where / / $(1=$ Not followed, $2=$ Home, $3=$ Doctor's office, $4=$ Confessional center, $5=$ Community Association Health Center, $6=$ Referral health center, $8=$ Medical clinic, $9=$ Community health center)

(Q23) Number of PNC /

(Q24) Prenatal check-up (PCU) / / $(1$ = Done, 2 = Not done $)$

2-General clinical examination:

(Q25) Size: / / (in meter)

(Q26) Weight: / $/(\mathrm{kg})$

(Q27) Conjunctivas / $/(1=$ colored, $2=$ pale $)$

(Q28) BP / / (mmhg)

(Q29) Temperature / $1{ }^{\circ} \mathrm{C}$

3-Obstetric examination:

(Q30) Uterine Height / / (centimeter)

(Q31) Painful uterine contraction (PUC)/ $/(1=$ Yes, $2=$ No $)$

(Q32) Fetal Heart Beat / / (beats / min)

(Q33) Presentation/ $/(1=$ cephalic, $2=$ breech, $3=$ transverse $)$

(Q34) Water bag / $/(1=$ intact, $2=$ broken $)$

(Q35) if broken, appearance of Amniotic fluid / / $(1$ = clear, 2 = yellowish, 3 = meconium)

(Q36) Pelvis / / $(1=$ Normal, $2=$ Limit, $3=$ Generally narrowed, $4=$ Asymmetrical)

(Q37) Birthing phase / / ( 1 = latency, 2 = Active, 3 = Not in labor $)$

$\mathrm{V}$-Indications for cesarean section:

(Q38) Indication of the caesarean section / $/(1=$ Acute fetal distress, $2=$ Proof of the beating cord, 3 = Feto-pelvic disproportion, $4=$ Seat in nulliparous, $5=$ Pre-rupture syndrome $6=$ PGN in labor, $7=$ Sherp grade II RPH, $8=\mathrm{Pla}$ centa previa hemorrhagic, $9=$ Irreducible dynamic dystocia, $10=$ Posterior face presentation $11=$ Face presentation, $12=$ Scar uterus on border pelvis, $13=$ Scar uterus on PGN, $14=$ Dynamic dystocia on scar uterus, $15=$ transverse presentation $16=$ presentation of bregma, $17=$ preeclampsia, $18=$ eclampsia, 19 $=$ twin pregnancy on scar uterus, $20=$ PRM on scar uterus, $21=$ twin pregnancy 
T1 in breech presentation, $22=$ pelvis immature, $23=$ Fetal macrosomia, $24=$ Short IIG / Scar uterus)

VI-Intraoperatively:

(Q39) Qualification of the operator / / $(1$ = Gynecologist, 2 = General practitioner, 3 = Internee)

(Q40) Type of skin incision: / / $(1$ = pfannenstiel, 2 = IMSO, $3=$ Joël Cohen)

(Q41) Type of hysterotomy / / $(1=$ Segmental, 2 = Corporeal, $3=$ Segmento-corporeal)

(Q42) Cutaneous incision and fetal extraction time / / in minutes

(Q43) Gesture associated with cesarean section / / $(1$ = None, 2 = Intrauterine device per-Cesarean section 3 = Hysterectomy, $4=$ Tubal ligation and resection, $5=$ Myomectomy, $6=$ Cystectomy, $7=$ Other $)$

(Q44) Total duration of the intervention / / in minutes

(Q45) Peroperative incident / / ( 1 = Bladder break-in, 2 = Intestinal perforation, 3 = Split line, 4 = Attack of vascular pedicle, $5=$ Nothing to report)

(Q46) Blood loss / / (in cubic centimeters)

VII-Anesthesia:

(Q47) Qualification of the anesthesiologist: / / $(1=$ anesthesiologist-resuscitator, 2 = Medical Assistant Nurse Anesthesiologist-Resuscitator)

(Q48) Type of anesthesia: / / $(1=\mathrm{GA}, 2$ = ALR, 3 = ALR converted to GA)

(Q49) Time between installation on operating table and skin incision: / in minutes

(Q50) Transfusion / / $(1=$ Yes, 2 = No $)$

(Q51) If transfusion why / / $(1$ = Hemorrhage, 2 = Anemia on pregnancy)

VIII-In post-operative:

(Q52) Complication / / $(1=$ None, 2 = Hemorrhagic, 3 = Infectious, $4=$ Anemia, $5=$ Thromboembolic, $6=$ Digestive, $7=$ Other $)$

(Q53) Duration of hospitalization / / in day (s)

IX-Newborn:

(Q54) Number /

(Q55) Sex / / ( 1 = Male, 2 = Female $)$

(Q56) Apgar 1st minute / / out of 10

(Q57) Apgar 5th minute / / out of 10

(Q58) Weight of Newborn / / in grams

(Q59) Size / / in centimeter

(Q60) Resuscitated newborn / $/(1=$ Yes, $2=$ No $)$

(Q61) Newborn evacuated to the neonatal service / / $(1=$ Yes, 2 = No $)$

(Q62) If yes, the reason for evacuation / $/(1=$ Newborn suffering, $2=$ Low birth weight, 3 = Macrosomy, $4=$ Neonatal infection, $5=$ Malformation, 6 $=$ Overdue delivery, $7=$ No referred, $8=$ Other $)$

(Q63) Fate of a newborn: / / $(1$ = Alive, 2 = Died $)$ 
(Q64) if death, the cause

X-Prognostic factors:

(Q65) Time between the teenager's departure from the source structure if reference/evacuation and the reception structure / $/$ in minutes

(Q66) Means of locomotion of Reference / Evacuation / / $(1=$ Ambulance, 2 = taxi, $3=$ Motorbike)

(Q67) Time between admission and indication of cesarean / / in minutes

(Q68) Time between indication and start of cesarean / / in minutes

(Q69) Fate of the patient after the cesarean / / $(1=$ Alive, 2 = Died $)$

(Q70) If death, the cause 